\title{
Usefulness of supernatant of pancreatic juice for genetic analysis of K-ras in diagnosis of pancreatic carcinoma
}

\author{
H. Watanabe, A. Ha, Y. Yamaguchi, K. Ohtsubo, Y. Wang, and N. Sawabu
}

Usefulness of K-ras analysis in pure pancreatic juice (PPJ) for the diagnosis of pancreatic carcinoma (PCa) was well known. To ascertain whether analysis of K-ras mutation at codon 12 in the supernatant of PPJ is more useful for the diagnosis of PCa than that in sediment, we analyzed K-ras mutation at codon 12 in DNA extract from not only the sediment but also the supernatant of PPJ and compared the results. PPJ was endoscopically collected from the 19 patients with $\mathrm{PCa}$ and 25 with chronic pancreatitis (CP). DNA was extracted from the supernatant and the sediment of PPJ, respectively. Mutant allele specific amplification (MASA) was performed for K-ras mutations analysis with the DNA extracts from these samples. As shown in Table, the incidence of K-ras mutations in the supernatant of PPJ was $89 \%$ (17 of 19) in patients with PCa and $28 \%$ (7 of 25) in patients with CP, whereas that in the sediment was 79\% (15 of 19) in patients with $\mathrm{PCa}$ and $20 \%$ (5 of 25) in patients with $\mathrm{CP}$. Although there was no significant difference in the incidence of K-ras mutations between supernatant and sediment, the positive rate of K-ras mutations was higher in the former. All of the patients with PCa showed K-ras mutations in either the supernatant or sediment of PPJ. Although there was no relation between the incidence of K-ras mutations in PPJ and the location and size of tumor, and clinical stage of carcinoma in the patients with PCa, two patients with clinical stage I disease showed K-ras mutations in the supernatant. These results suggest that the positive rate of K-ras mutations in the supernatant is not less than that in the sediment, and simultaneous analysis of K-ras mutations in the supernatant and sediment of PPJ enhances the genetic diagnosis of PCa.

Table Comparison of the incidence of K-ras mutations at codon 12 in supernatant or sediment of pure pancreatic juice from patients with pancreatic carcinoma and chronic pancreatitis detected by mutant allele specific amplification

\begin{tabular}{|c|c|c|c|}
\hline \multirow[b]{2}{*}{ Disease } & \multirow{2}{*}{$\begin{array}{l}\text { No. of cases } \\
\text { examined }\end{array}$} & \multicolumn{2}{|c|}{ No. (\%) of K-ras mutation-positve cases by MASA } \\
\hline & & Supernatant & Sediment \\
\hline Pancreatic carcinoma & 19 & $17(89)$ & $15(79)$ \\
\hline Chronic pancreatitis & 25 & $7(28)$ & $5(20)$ \\
\hline
\end{tabular}

* no significant difference by chi-square test 